\title{
Obtaining Better Non-Dominated Sets Using Volume Dominance
}

\author{
Khoi Le, Dario Landa-Silva \\ School of Computer Science and Information Technology \\ University of Nottingham, UK \\ http: / / www.asap.cs.nott.ac.uk \\ E-mail: kxl@cs.nott.ac.uk,jds@cs.nott.ac.uk
}

\begin{abstract}
An important goal in multiobjective optimisation is to find a good set of non-dominated solutions that is both well-distributed and well-converged. Most multiobjective optimisation algorithms use the conventional Pareto dominance relationship. Over recent years, new approaches for the dominance relationship such as relaxed Pareto dominance, have been proposed. The interest in relaxed forms of Pareto dominance has been increasing due to their capability to find extreme values in the objective space. This paper conducts a short review on relaxed forms of Pareto dominance in the literature. A new form of relaxed Pareto dominance, called volume dominance, is presented afterward. The results based on comparison between volume dominance and Pareto dominance using SEAMO2, SPEA2 and NSGA2 show that the proposed volume dominance is capable of obtaining a better and smoother trade-off front, and it is more robust than Pareto dominance.
\end{abstract}

\section{INTRODUCTION}

Many real-world optimisation problems are multiobjective and the interest on heuristic techniques to solve these kind of problems has increased considerably over the last decade or so. Confronted with a multiobjective optimisation problem, a decision-maker should find an appropriate solution that represents a good compromise between the several possibly conflicting objectives. It is not easy to find such one solution because there are usually more than one good tradeoff solution. In Pareto based multiobjective optimisation, a set of non-dominated solutions, also known as Pareto front, is sought so that the decision-maker can select the most appropriate one. Using Pareto dominance, a solution $x$ is said to be non-dominated if there is no other solution that is better than $x$ at least one objective and as good as $x$ in other objectives.

Evolutionary algorithms seem especially suitable to deal with Pareto based multiobjective optimisation problems because they can produce multiple promising solutions in a single run. Consequently, they can evolve a population of solutions towards the Pareto front. A good multiobjective evolutionary algorithm (MOEA) should be able to obtain Pareto fronts that are both well-distributed and wellconverged. Two issues when designing a MOEA are to decide how solutions in the population are evolved and to decide how to establish superiority between solutions in the population (i.e. how to compare solution fitness in a multiobjective sense). With respect to the first issue, a number of techniques have been investigated to 'push' solutions towards the desired part of the tradeoff surface. For example, directed weighted vectors, restricted mating, archiving elite solutions, clustering/crowding, fitness sharing, specialised operators, etc. have been proposed to improve the distribution and the convergence of the population towards the Pareto front [1], [2], [3]. However, we believe that the latter issue of assigning fitness to solutions in the multiobjective context has received less attention than it deserves.

For assigning fitness to solutions, most modern MOEAs adopt the conventional Pareto dominance relationship. There are few papers that propose different types of dominance relationship such as $\alpha$-dominance, $\epsilon$-dominance, E-Pareto dominance and fuzzy dominance (details in section 2). These variations of dominance aim to find solutions in difficult areas (like the extremes of the tradeoff surface) or attempt to combine convergence and diversity in order to achieve better Pareto front in difficult problems. These variations of Pareto dominance, called relaxed Pareto dominance here, apply some transferring functions to the objective values before comparing the solutions using Pareto dominance. It has been shown that relaxed Pareto dominance helps to obtain better quality Pareto fronts in some problems (e.g. [4], [5], [6]). In this paper, we propose a new form of relaxed Pareto dominance, called volume dominance. The volume dominance relationship proposed here compares two solutions with respect to the volume that each solution dominates and also taking into consideration the volume that both solutions dominate simultaneously (shared dominated volume). Then we compute a relative dominated volume to establish preference in the multiobjective context. We compare the performance of some well-known MOEAs when using the proposed volume dominance and the conventional Pareto dominance. We use the multiple knapsack problem in our experiments because benchmark results are available for this problem. We also show how our proposed volume dominance could be adapted for use in other MOEAs.

Section 2 presents a short literature review of Pareto dominance and relaxed forms of Pareto dominance. Section 3 describes our proposed form of relaxed Pareto dominance, volume dominance. Section 4 describes our experiments to compare the impact of using volume dominance against that of using Pareto dominance on three well-known MOEAs from the literature. We discuss our results in Section 5 while section 6 gives conclusions and summarises future work. 


\section{LiterATURE REVIEW}

Without loss of generality, we consider the multiobjective optimisation problem with $m$ objectives to be maximised.

$$
\text { maximise } \quad\left\{f_{1}(\vec{x}), f_{2}(\vec{x}), \ldots, f_{m}(\vec{x})\right\}
$$

subject to the decision vector $\vec{x}=\left(x_{1}, x_{2}, \ldots, x_{n}\right)^{T}$ belongs to the feasible region $S$ formed by constraint conditions. Then, the objective vector of $\vec{x}$ is

$$
\vec{f}(\vec{x})=\left(f_{1}(\vec{x}), f_{2}(\vec{x}), \ldots, f_{m}(\vec{x})\right)
$$

\section{A. Pareto Dominance}

The concept of Pareto dominance or Pareto optimum was proposed by Vilfredo Pareto in 1896 [7]. In the last two decades or so, Pareto dominance has been widely adopted as the technique to compare the fitness of solutions in multiobjective optimisation. In Pareto dominance, a solution $\vec{x}$ is considered to be better than a solution $\overrightarrow{x^{*}}$ if and only if the objective vector of $\vec{x}$ dominates the objective vector of $\overrightarrow{x^{*}}$. More formally:

Pareto Dominance. A solution $\vec{x} \in S$ dominates a solution $\overrightarrow{x^{*}} \in S\left(\vec{x} \succ \overrightarrow{x^{*}}\right)$ if and only if $\vec{x}$ is not worse than $\overrightarrow{x^{*}}$ in all objectives $\left(f_{i}(\vec{x}) \geq f_{i}\left(\overrightarrow{x^{*}}\right) \forall i=1, \ldots, m\right)$ and $\vec{x}$ is better than $\overrightarrow{x^{*}}$ in at least one objective $\left(f_{i}(\vec{x})>f_{i}\left(\overrightarrow{x^{*}}\right)\right.$ for at least one $i=1, \ldots, m$ ).

Within Pareto dominance, we can distinguish between strong dominance and weak dominance [6] or strict dominance and loose dominance respectively [8].

Strong dominance. A solution $\vec{x}$ strongly dominates a solution $\overrightarrow{x^{*}}$ if $\vec{x}$ is better than $\overrightarrow{x^{*}}$ in all objectives.

Weak dominance. A solution $\vec{x}$ weakly dominates a solution $\overrightarrow{x^{*}}$ if $\vec{x}$ is better than $\overrightarrow{x^{*}}$ at least one of the objectives and is as good as $\overrightarrow{x^{*}}$ in all other objectives.

In both of the above types of Pareto dominance, if neither $\vec{x}$ dominates $\overrightarrow{x^{*}}$ nor $\overrightarrow{x^{*}}$ dominates $\vec{x}$, then both solutions are said to be incomparable or non-dominated. In this case, no solution is clearly preferred over the other one. The set $F$ consisting of all non-dominated solutions $\vec{x} \in S$ is called the Pareto-optimal front with respect to $S$. A solution $\vec{x} \in F$ if there is no solution $\overrightarrow{x^{*}} \in S$ that dominates $\vec{x}$.

\section{B. Relaxed Pareto Dominance}

Relaxed forms of Pareto dominance have been recently proposed as a way to improve the performance of multiobjective optimisers. In general, relaxed Pareto dominance allows a solution $\vec{x}$ to dominate another solution $\overrightarrow{x^{*}}$ for which $\vec{x}$ and $\overrightarrow{x^{*}}$ are Pareto non-dominated solutions or even $\vec{x}$ is Pareto-dominated by $\overrightarrow{x^{*}}$, examples of relaxed dominance are described below.

The first form of relaxed Pareto dominance was that by $\mathrm{Yu}$ in 1974 who proposed a structure of domination over the objective space to explore the geometry of the set of all non-dominated solutions [9]. Two new concepts of cone convexity and cone extreme point were introduced to study decisions problems on polar cones and polyhedral cones. However, it is not until recently that there is an increasing interest in relaxed Pareto dominance within the multiobjective optimisation community.

In 2001, Kokolo et al. introduced $\alpha$-dominance to deal with what they call dominance resistant solutions, i.e. solutions that are fairly inferior qualitatively but for which dominating solutions are scarely found [10]. The main idea of $\alpha$-dominance is to set up upper and lower bounds of tradeoffs between objectives. In $\alpha$-dominance, small detriments in one objective are considered acceptable if it leads to a noticeable improvement in other objectives. For the formal definition of $\alpha$-dominance see [10].

Laumanns et al. proposed a slightly different but simpler form of relaxed dominance called $\epsilon$-dominance which seeks to combine diversity and convergence in one criterion [4]. A solution with objective vector $f$ is said to $\epsilon$-dominate a solution with objective vector $g$ if and only if $(1+\epsilon) \cdot f_{i} \geq$ $g_{i} \forall i \in 1, \ldots, m$. The main difference between $\epsilon$-dominance and $\alpha$-dominance is that $\epsilon$-dominance allows some Paretodominated solutions (e.g. $\vec{x} \succ \overrightarrow{x^{*}}$ ) to actually become preferred (e.g. $\overrightarrow{x^{*}} \epsilon$-dominates $\vec{x}$ ) which is not the case in $\alpha$-dominance.

Jin and Wong proposed extended Pareto dominance (EPareto) in their Adaptive Rectangle Archiving algorithm [11]. Extended Pareto dominance is quite similar to $\epsilon$-dominance in the sense that both apply some sort of transferring functions to the objective vector before comparing them using Pareto dominance. In extended Pareto dominance, $\vec{x}$ E-dominates $\overrightarrow{x^{*}}$ for some transferring function, $F U N$, and a constant vector $e(>0)$ if and only if $\forall i \in 1, \ldots, m$ $F U N\left(f_{i}(\vec{x})\right) \geq F U N\left(f_{i}\left(\overrightarrow{x^{*}}\right)\right)-e_{i}$. Jin and Wong compared E-dominance to $\epsilon$-dominance and the conventional Pareto dominance and stated that E-dominance becomes $\epsilon$ dominance as $F U N\left(f_{i}(\vec{x})\right)=\ln \left(f_{i}(\vec{x})\right)$ and $e_{i}=\ln (1+\epsilon)$ and Pareto dominance as $F U N\left(f_{i}(\vec{x})\right)=f_{i}(\vec{x})$ and $e_{i}=0$.

Several researchers have investigated the fuzzification of Pareto dominance. In fuzzy-Pareto-dominance proposed by Koppen et al. [5], $\vec{x}$ dominates $\overrightarrow{x^{*}}$ by degree $\mu_{a}$ with

$$
\mu_{a}\left(\vec{x}, \overrightarrow{x^{*}}\right)=\frac{\prod_{i=1}^{m} \min \left(f_{i}(\vec{x}), f_{i}\left(\overrightarrow{x^{*}}\right)\right)}{\prod_{i=1}^{m} f_{i}\left(\overrightarrow{x^{*}}\right)}
$$

and $\vec{x}$ is dominated by $\overrightarrow{x^{*}}$ at degree $\mu_{p}$ with

$$
\mu_{p}\left(\vec{x}, \overrightarrow{x^{*}}\right)=\frac{\prod_{i=1}^{m} \min \left(f_{i}(\vec{x}), f_{i}\left(\overrightarrow{x^{*}}\right)\right)}{\prod_{i=1}^{m} f_{i}(\vec{x})}
$$

This fuzzy form of Pareto dominance becomes the conventional Pareto dominance (for $\vec{x} \succ \overrightarrow{x^{*}}$ ) when $\mu_{a}\left(\vec{x}, \overrightarrow{x^{*}}\right)=1$ and $\mu_{p}\left(\overrightarrow{x^{*}}, \vec{x}\right)=1$, but $\mu_{p}\left(\vec{x}, \overrightarrow{x^{*}}\right)<1$ and $\mu_{a}\left(\overrightarrow{x^{*}}, \vec{x}\right)<1$. Koppen et al. applied fuzzy-Pareto-dominance to deal with what they call the Pareto-Box problem, determining the expectation value for the size of the Pareto set of $m$ points in an $n$-dimensional space.

Peng et al. proposed a different concept for fuzzy dominance based on the credibility distribution of fuzzy variables [12]. Here, $\xi$ and $\eta$ are two fuzzy variables with the credibility distribution $\Phi(x)$ and $\Psi(x)$ respectively, where $\Phi^{(k)}(x)=\int_{-\infty}^{x} \Phi^{(k-1)}(t) d t$ and $\Psi^{(k)}(x)=$ 
$\int_{-\infty}^{x} \Psi^{(k-1)}(t) d t \quad k=2,3, \ldots$ and $\Phi^{(1)}(x)=\Phi(x)$ and $\Psi^{(1)}(x)=\Psi(x)$. Then, it is said that $\xi k$-Order Fuzzy Dominates $\eta$ if and only if $\Phi^{(k)}(x) \leq \Psi^{(k)}(x) \forall x \in \Re$. For more discussion on credibility distribution fuzzy dominance see [12].

Burke and Landa-Silva proposed another form of relaxed dominance using a gaining factor [6]. For a 2-objective maximisation problem, $\vec{x}$ dominates $\overrightarrow{x^{*}}$ if $f_{2}\left(\overrightarrow{x^{*}}\right)<f_{2}(\vec{x}) \cdot(1+$ gain) where gain $=\left(f_{1}(\vec{x})-f_{1}\left(\overrightarrow{x^{*}}\right)\right) / f_{1}(\vec{x})$ which is equivalent to the following relation:

$$
\frac{f_{1}\left(\overrightarrow{x^{*}}\right)}{f_{1}(\vec{x})}+\frac{f_{2}\left(\overrightarrow{x^{*}}\right)}{f_{2}(\vec{x})}<2
$$

For $m$ objectives maximisation problem, $\vec{x}$ dominates $\overrightarrow{x^{*}}$ if

$$
\frac{f_{1}\left(\overrightarrow{x^{*}}\right)}{f_{1}(\vec{x})}+\frac{f_{2}\left(\overrightarrow{x^{*}}\right)}{f_{2}(\vec{x})}+\ldots+\frac{f_{m}\left(\overrightarrow{x^{*}}\right)}{f_{m}(\vec{x})}<m
$$

They showed that the performance of two multiobjective algorithms was improved by using relaxed dominance when solving a highly constrained combinatorial optimisation problem.

\section{Volume Dominance}

All the above forms of relaxed dominance, like the conventional Pareto dominance, are based on comparing the objective vectors of solutions in one way or another. We propose a new form of relaxed Pareto dominance, called volume dominance which is based on a different concept: comparing the dominated volumes in the objective space between two solutions $\vec{x}$ and $\overrightarrow{x^{*}}$. The dominated volume of $\vec{x}$ is defined as the region $R$ for which all feasible solutions in $R$ are dominated by $\vec{x}$. In order to determine the dominated volume of solution $\vec{x}$, we need to define a reference point $\vec{r}$ in the objective space corresponding to a solution $x^{-}$. The solution $x^{-}$is dominated by all other solutions which objective vectors are also in $R$. Hence, the formula to calculate the dominated volume of $\vec{x}$ with respect to the reference point $\vec{r}=\left(r_{1}, r_{2}, \ldots, r_{m}\right)$ is defined as follows:

$$
V_{\vec{x}}=\prod_{i=1}^{m}\left(f_{i}(\vec{x})-r_{i}\right)
$$

Then the dominated volumes of $\vec{x}$ and $\overrightarrow{x^{*}}$ are compared to establish the dominance relationship between $\vec{x}$ and $\overrightarrow{x^{*}}$.

Our volume dominance relationship is not based on directly comparing the two dominated volumes. Instead, it is based on the relative dominated volume. The relative dominated volume is the volume of the region that is dominated by both $\vec{x}$ and $\overrightarrow{x^{*}}$, called shared dominated volume. The shared dominated volume is defined as follows:

$$
S V_{\vec{x}, \overrightarrow{x^{*}}}=\prod_{i=1}^{m}\left(\min \left(f_{i}(\vec{x}), f_{i}\left(\overrightarrow{x^{*}}\right)\right)-r_{i}\right)
$$

The volume dominance relationship of $\vec{x}$ and $\overrightarrow{x^{*}}$ is then established by comparing the dominated volumes $V_{\vec{x}}$ and $V_{\overrightarrow{x^{*}}}$ to the shared dominated volume $S V_{\vec{x}, x^{*}}$. Then, we say that $\overrightarrow{x^{*}}$ is volume-dominated by $\vec{x}\left(\overrightarrow{x^{*}} \prec_{V} \vec{x}\right)$ for some ratios $r S V$ if either:

- $V_{\overrightarrow{x^{*}}}=S V_{\vec{x}, \overrightarrow{x^{*}}}$ and $V_{\vec{x}}>S V_{\vec{x}, \overrightarrow{x^{*}}}$ or

- $V_{\vec{x}}>V_{\overrightarrow{x^{*}}}>S V_{\vec{x}, \overrightarrow{x^{*}}}$ and $r_{\vec{x}, \overrightarrow{x^{*}}}=\frac{V_{\vec{x}}-V_{\overrightarrow{x^{*}}}}{S V_{\vec{x}, x^{*}}}>r S V$

The $r S V$ value sets the target shared volume ratio. A small $r S V$ indicates that a small difference between the dominated volumes of $\vec{x}$ and $\overrightarrow{x^{*}}$ (w.r.t $S V_{\vec{x}, \overrightarrow{x^{*}}}$ ) is enough to discriminate between $\vec{x}$ and $\overrightarrow{x^{*}}$. Here, we experiment with different values of $r S V$.

At the first sight, the above volume dominance relationship seems to be very similar to the $\mathcal{S}$ metric proposed in [17]. However the underlying principle is different. The $\mathcal{S}$ metric measures the size of the volume covered by a set of nondominated solutions to determine how good that set is in comparison with another set of non-dominated solutions. The volume dominance deploys the size of volume covered by a single solution to establish the dominance relationship between any two solutions. Moreover, when using the $\mathcal{S}$ metric the volumes covered by the two sets are compared directly while in the volume dominance the shared dominated volume is also considered.

Figure 1 illustrates the volume dominance relationship between $\vec{x}$ and $\overrightarrow{x^{*}}$ in a 2-objective space with the reference point $\vec{r}$ in the origin $O(0,0)$. In this example, $\vec{x}$ volumedominates $\overrightarrow{x^{*}}$ for some values of $r S V$.

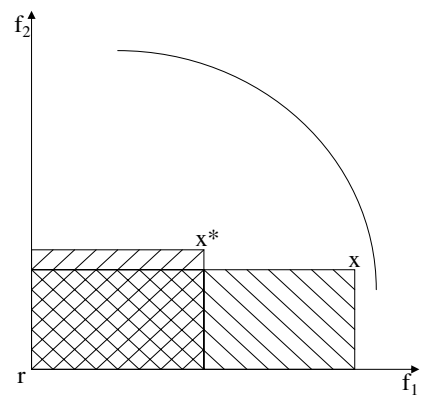

Fig. 1. Volume Dominance - A Form of Relaxed Dominance

The volume dominance proposed in this paper clearly covers Pareto dominance:

Proof: if $\vec{x}$ Pareto-dominates $\overrightarrow{x^{*}}\left(\vec{x} \succ \overrightarrow{x^{*}}\right)$ i.e. $f_{i}(\vec{x}) \geq$ $f_{i}\left(\overrightarrow{x^{*}}\right) \forall i=1, \ldots, m$ and $f_{i}(\vec{x})>f_{i}\left(\overrightarrow{x^{*}}\right)$ for at least one $i=1, \ldots, m$. The shared dominated volume:

$$
\begin{aligned}
S V_{\vec{x}, \overrightarrow{x^{*}}} & =\prod_{i=1}^{m}\left(\min \left(f_{i}(\vec{x}), f_{i}\left(\overrightarrow{x^{*}}\right)\right)-r_{i}\right) \\
& =\prod_{i=1}^{m}\left(f_{i}\left(\overrightarrow{x^{*}}\right)-r_{i}\right)=V_{\overrightarrow{x^{*}}}
\end{aligned}
$$

As $f_{i}(\vec{x}) \geq f_{i}\left(\overrightarrow{x^{*}}\right) \forall i=1, \ldots, m$ and $f_{i}(\vec{x})>f_{i}\left(\overrightarrow{x^{*}}\right)$ for at least one $i=1, \ldots, m$, then $V_{\vec{x}}>V_{\overrightarrow{x^{*}}}$ i.e. $V_{\vec{x}}>S V_{\vec{x}, \overrightarrow{x^{*}}}$. Therefore, $\vec{x}$ volume-dominates $\overrightarrow{x^{*}}\left(\vec{x} \succ_{V} \overrightarrow{x^{*}}\right)$.

It is noted that the proposed volume dominance contains a normalisation element which prevents bias in some directions in cases with non-commensurable objective functions. 
Proof: Suppose it is required to normalise each objective function using $\vec{n}=\left\{n_{i} \mid i: 1 \ldots m\right\}$ then

$$
\begin{aligned}
V_{\vec{x}}^{N} & =\prod_{i=1}^{m}\left(\frac{f_{i}(\vec{x})}{n_{i}}-\frac{r_{i}}{n_{i}}\right)=\prod_{i=1}^{m} \frac{f_{i}(\vec{x})-r_{i}}{n_{i}} \\
& =\frac{\prod_{i=1}^{m}\left(f_{i}(\vec{x})-r_{i}\right)}{\prod_{i=1}^{m} n_{i}}=\frac{V_{\vec{x}}}{\prod_{i=1}^{m} n_{i}} \\
S V_{\vec{x}, x^{*}}^{N} & =\prod_{i=1}^{m}\left(\min \left(\frac{f_{i}(\vec{x})}{n_{i}}, \frac{f_{i}\left(\overrightarrow{x^{*}}\right)}{n_{i}}\right)-\frac{r_{i}}{n_{i}}\right) \\
& =\prod_{i=1}^{m} \frac{\min \left(f_{i}(\vec{x}), f_{i}\left(\overrightarrow{x^{*}}\right)\right)-r_{i}}{n_{i}} \\
& =\frac{\prod_{i=1}^{m}\left(\min \left(f_{i}(\vec{x}), f_{i}\left(\overrightarrow{x^{*}}\right)\right)-r_{i}\right)}{\prod_{i=1}^{m} n_{i}} \\
& =\frac{S V_{\vec{x}, \overrightarrow{x^{*}}}}{\prod_{i=1}^{m} n_{i}} \\
r_{\vec{x}, \overrightarrow{x^{*}}}^{N} & =\frac{V_{\vec{x}}^{N}-V_{\overrightarrow{x^{*}}}^{N}}{S V_{\vec{x}, \overrightarrow{x^{*}}}^{N}}=\frac{\overrightarrow{\prod_{i=1}^{m} n_{i}}-\frac{V_{\overrightarrow{x *}}}{\prod_{i=1}^{m} n_{i}}}{\prod_{\overrightarrow{\vec{x}}, \overrightarrow{x^{*}}}^{m}} \\
& =\frac{V_{\vec{x}}-V_{\overrightarrow{x^{*}}}}{S V_{\vec{x}, \overrightarrow{x^{*}}}}=r_{\vec{x}, x^{*}}
\end{aligned}
$$

There is a crucial difference between the proposed volume dominance and other dominance relationships including conventional Pareto dominance and other forms of relaxed dominance proposed in the literature. In order to decide dominance between solutions, volume dominance takes all objectives into consideration at once by combining them into a single unit vector rather than directly comparing each objective in turn as it happens in other dominance relationships. In this way, volume dominance seeks to evaluate the whole objective vectors to somehow compensate improvement and detriment between objectives.

\section{EXPERIMENTAL DESIGN}

Most relaxed forms of dominance presented in the literature aim to reach and maintain extreme points in the objective space or points that are difficult to reach and maintain with Pareto dominance while others aim to combine diversity and convergence into a single criterion when discriminating between solutions. These relaxed dominances have been proposed as an integral part of specific multiobjective algorithms, see [4], [5], [6], [9], [10], [12]. To the best of our knowledge, none of these forms of relaxed dominance has been tested on different multiobjective optimisers and using a benchmark problem in order to compare it to the conventional Pareto dominance. We aim to address this issue in this paper.

The volume dominance proposed here was conceived in our work tackling the QMC nurse scheduling problem, a highly constrained personnel scheduling problem [13]. In this problem, there are a number of extreme points in the tradeoff surface and it is very difficult to find feasible solutions due to the constrained nature of the problem. Our work on the QMC nurse scheduling problem is still ongoing but preliminary results showed that volume dominance helps multiobjective optimisers to obtain better results.

In this paper, we present experimental results showing that volume dominance works well on different multiobjective evolutionary algorithms such as SEAMO2 [14], SPEA2 [15] and NSGA2 [16] when solving the multiple knapsack problem [17], a well-known benchmark multiobjective combinatorial optimisation problem. SEAMO2 uses a steady-state population and a simple elitist replacement strategy. The algorithm chooses each member of the population, in turn, to be the first parent and a second parent is chosen at random. Offspring is produced by applying cycle crossover on the two parents followed by a single mutation. If the offspring's objective vector improves on any best-so-far objective function, it replaces one of the parents and the objective's best-so-far is updated. Otherwise, if the offspring dominates one of the parents, it replaces that parent (unless it is a duplicate, then the offspring is deleted). If neither the offspring dominates the parents nor the parents dominate the offspring, the offspring replaces a random solution in the population that the offspring dominates. SPEA2 uses a fixed size archive to store non-dominated solutions in addition to a population. SPEA2 deploys a fine-grained fitness assignment strategy which takes for each individual into account how many individuals it dominates and it is dominated by. A nearest neighbour density estimation for environmental selection is used to deal with two situations: when either the archive is too small or too large. The best dominated individuals in the previous archive and the population are copied to the new archive in the fist case. In the latter situation, non-dominated individuals in the archive are iteratively removed until the archive's size is not exceeded. The removal of non-dominated individuals from the archive is carefully managed by using an archive truncation method that guarantees the preservation of boundary solutions. NSGA2 uses a fast non-dominated sorting algorithm to classify a population into different nondomination levels. NSGA2 also uses a crowding technique based on the density of solutions surrounding a particular solution to preserve the diversity of the population. SEAMO2 is implemented according to its description in [14] whereas SPEA2 and NSGA2 are obtained from the PISA [18] platform. Parameter settings for tackling the multiple knapsack problem with SPEA2 and NSGA2 were kindly provided by Marco Laumanns by means of email based discussions. Then, in our experiments we replace the conventional Pareto dominance with our proposed volume dominance and analyse the impact on the performance of these three algorithms.

We use the 750 items and 4 objectives instance of the knapsack problem proposed in [17]. In order to investigate the proposed volume dominance, we carry out short and long runs with medium and large population sizes and using different values of $r S V$. Each short run uses 175,000 fitness evaluations and each long run uses 672,000 fitness evaluations. The values used of population size are 250 and 350 individuals. For the volume dominance we use 6 
different values of $r S V, 0.05,0.10,0.15,0.20,0.25$ and 0.30 . The reference point for volume dominance is chosen as the origin in the objective space. Below, we summarise and discuss the results from 50 independent short runs and 30 independent long runs.

We use two metrics to evaluate the non-dominated fronts produced by the volume dominance approach and the conventional Pareto dominance approach. These two metrics, size of the space covered $\mathcal{S}$ and coverage of two sets $\mathcal{C}$, were proposed in [17]. The $\mathcal{S}$ hypervolume metric, which measures the overall size of objective space covered by all the non-dominated solutions, is scaled as the percentage of the volume created by the origin and the reference point (41656, 40363, 41905, 41744) which is the sum of profits of all items in each objective. The boxplots in Figure 3, 4, 7 present the distribution of the $\mathcal{S}$ hypervolume metric. The vertical axes of the boxplots measure the percentage of non-dominated objective space. The horizontal axes present Pareto dominance (PD) and volume dominance (VD) with different $r S V$ ratios. The average values for the coverage metric $\mathcal{C}$, which compares the dominance of the Pareto front obtained by one opmitisation technique to that obtained by another optimisation technique, are given in Table I, II, III. The standard deviations are given in brackets.

\section{RESUlTS AND DisCUSSION}

With respect to the coverage metric $\mathcal{C}$, the performance of SEAMO2 with the proposed volume dominance and the conventional Pareto dominance is quite similar (Table I). In Table I, the column labels present the population size and the number of generations (e.g. 250-700 indicates the population size of 250 individuals evolved for 700 generations) and the row labels VDx refer volume dominance using $r S V=$ $x / 100$. However, with respect to the hypervolume metric $\mathcal{S}$ (Figure 3), volume dominance in SEAMO2 suffers from using lower ratios $r S V$ especially in longer runs. Using higher ratios $r S V$, volume dominance obtains competitive results compared to the conventional Pareto dominance, in both $\mathcal{S}$ and $\mathcal{C}$ metrics.
TABLE I

AVERAGE VALUES (STANDARD DEVIATION) OF coverage of two sets FOR $\mathrm{SEAMO} 2 \mathcal{C}(\mathrm{A} \succ \mathrm{B})$

\begin{tabular}{|l|l|c|c|c|c|}
\hline \multicolumn{7}{|c|}{ SEAMO2 $\mathcal{C}(\mathrm{A} \succ \mathrm{B})$} \\
\hline \multicolumn{2}{|c|}{ Dominance } & \multicolumn{5}{|c|}{} \\
\hline A & B & $250-700$ & $350-500$ & $250-2688$ & $350-1920$ \\
\hline PD & VD5 & $20.6(34.3)$ & $23.8(27.4)$ & $1.7(4.8)$ & $15.5(23.8)$ \\
& VD10 & $21.7(24.5)$ & $21.9(25.0)$ & $8.0(15.7)$ & $18.4(22.6)$ \\
& VD15 & $25.6(33.7)$ & $20.5(28.5)$ & $11.9(14.8)$ & $16.5(20.1)$ \\
& VD20 & $14.9(23.0)$ & $25.4(25.9)$ & $12.1(14.1)$ & $19.7(23.4)$ \\
& VD25 & $18.4(26.3)$ & $27.4(27.8)$ & $17.2(17.3)$ & $19.8(24.9)$ \\
& VD30 & $17.8(21.6)$ & $26.7(27.7)$ & $15.1(17.4)$ & $14.8(18.6)$ \\
\hline VD5 & PD & $18.0(26.9)$ & $23.1(28.6)$ & $20.0(17.8)$ & $16.3(16.4)$ \\
VD10 & & $17.0(24.7)$ & $22.9(29.2)$ & $17.6(16.8)$ & $14.2(19.6)$ \\
VD15 & & $23.4(30.3)$ & $21.9(25.6)$ & $18.7(19.6)$ & $13.9(20.2)$ \\
VD20 & & $23.6(22.8)$ & $17.2(25.1)$ & $14.1(17.2)$ & $14.2(17.2)$ \\
VD25 & & $23.3(26.7)$ & $15.9(22.0)$ & $11.8(13.4)$ & $10.1(13.0)$ \\
VD30 & & $20.3(24.9)$ & $16.4(23.5)$ & $16.4(19.3)$ & $21.0(21.8)$ \\
\hline
\end{tabular}

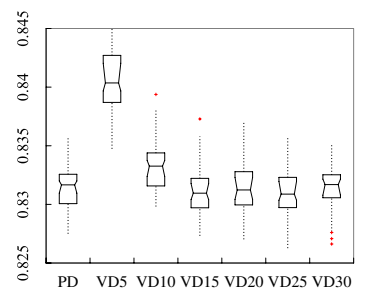

(a) $250-700$

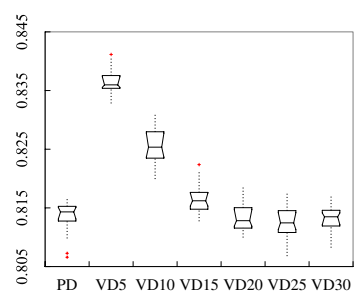

(c) $250-2688$

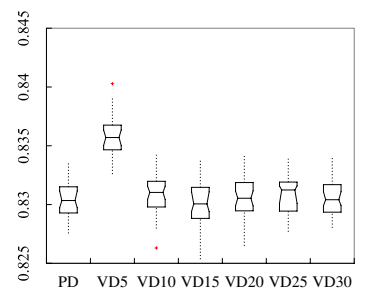

(b) $350-500$

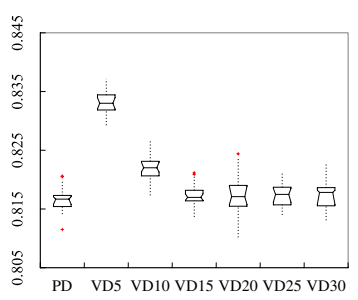

(d) $350-1920$
Fig. 3. Performance of PD and VD on SEAMO2 for 4-objective knapsack problem based on size of the space covered metric $\mathcal{S}$. Each graph label indicates the population size - the number of generations

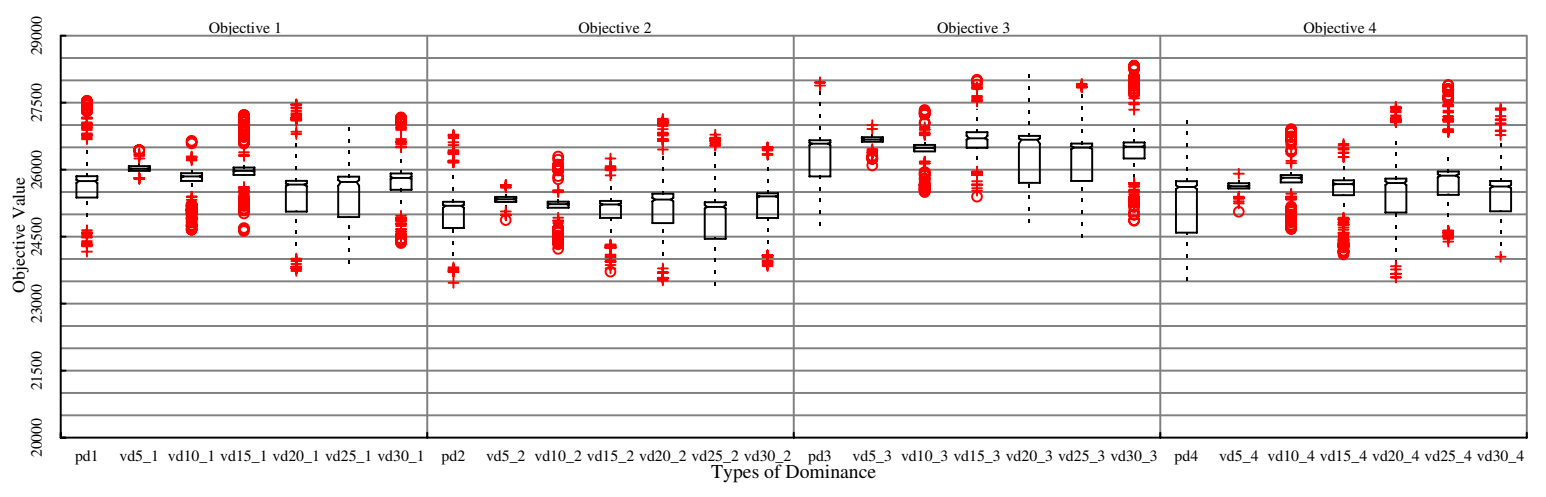

Fig. 2. The distribution of objective values obtained when using conventional Pareto dominance (PD) and volume dominance (VD) on SEAMO2 for 4-objective knapsack problem 
A closer look at the values of individual objectives in the final non-dominated sets, suggests that SEAMO2 with the conventional Pareto dominance has already obtained good extreme points in the objective space. The sets of extreme points obtained with volume dominance using high ratios $r S V$ are quite similar to the ones achieved with Pareto dominance. It seems that lower ratios $r S V$ are less able to produce extreme points. However, volume dominance with low ratios $r S V$ is better in pushing the set of the final nondominated solutions towards the Pareto front. In other words, the range of objective values is much better when using volume dominance with lower ratios $r S V$.

Figure 2 presents the distribution of each objective in the objective space for one particular run using SEAMO2 with population size of 250 in a long run. The vertical axis represents the objective value while the horizontal axis represents each objective with different types of dominance. For example, in the horizontal axis, pd1 stands for objective 1 of Pareto dominance, and vd5_1 stands for objective 1 of volume dominance using $r S V=0.05$.

We predicted that the search strategy in SEAMO2, which outperforms two well-known algorithms SPEA2 and NSGA2 [14], should be able to obtain good extreme values in the objective space but its trade-off front shows a lot of variation. Figure 2 provides evidence to support this, we can see that there is a large number of outlier values in the boxplots for each objective when using Pareto dominance (PD). Volume dominance using low ratios $r S V$ is less able to find outlier values. However, it is able to obtain a smoother tradeoff front with significantly less variation. Figure 2 shows that the boxplots for volume dominance using ratios $r S V$ of $0.05,0.10$ and 0.15 (VD5, VD10 and VD15 respectively) are much smaller than the ones for Pareto dominance in all 4 objectives. Volume dominance using higher ratios obtains similar distribution of objective values as those achieved by the conventional Pareto dominance.

In general, for SEAMO2 volume dominance is not capable of finding good extreme objective values as it is the case for Pareto dominance but it obtains a smoother trade-off front, especially when using lower ratios $r S V$. Furthermore, volume dominance helps SEAMO2 to push the trade-off front forward as a whole without bias on a particular objective.

Volume dominance when deployed in SPEA2 and NSGA2 using low ratios $r S V$ clearly outperforms Pareto dominance with respect to the $\mathcal{C}$ metric as seen in Tables II and III). For example, in Table II, for the run with population size of 250 and using 700 generations (250-700 column), none of the non-dominated solutions produced using Pareto dominance dominates solutions produced using volume dominance for the case when $r S V=0.05$ (PD VD5 0(0)). On the other hand, we can see 6 rows below in the table that $78.1 \%$ of the non-dominated solutions produced using volume dominance for the case when $r S V=0.05$ dominate solutions produced using Pareto dominance with a standard deviation of $7.4 \%$ based on 50 independent runs (VD5 PD 78.1(7.4)). However, as in SEAMO2, the hypervolume of the final non-dominated set obtained by deploying volume dominance with low ratios $r S V$ in SPEA2 and NSGA2 is worse than the one obtained by deploying Pareto dominance, but not as bad as in SEAMO2 (Figures 4 and 7).

As in SEAMO2, we also analyse the distribution of each objective value in the objective space when using SPEA2 and NSGA2 with the two types of dominance. Figures 5 and 6 present results for a particular run using SPEA2 and NSGA2 respectively. As it is shown, volume dominance using low ratios $r S V$, deployed in SPEA2 and NSGA2, is slightly worse in obtaining extreme objective values than Pareto dominance. However volume dominance is better in pushing the tradeoff front forward in all objectives and obtaining a smoother trade-off front. Figure 5 and 6 show a better range and a smaller size of the boxplot for volume dominance using $r S V=0.05$ than for Pareto dominance.

Based on our results with respect to the size of the space covered $\mathcal{S}$, coverage of two sets $\mathcal{C}$ and the distribution of the trade-off front, we suggest that the $r S V$ ratio should be in the range of 0.15 to 0.20 for SEAMO2 and around 0.10 for SPEA2 and NSGA2.

TABLE II

AVERAGE VALUES (STANDARD DEVIATION) OF coverage of two sets FOR SPEA2 $\mathcal{C}(\mathrm{A} \succ \mathrm{B})$

\begin{tabular}{|c|c|c|c|c|c|}
\hline \multicolumn{6}{|c|}{ SPEA2 $\mathcal{C}(\mathrm{A} \succ \mathrm{B})$} \\
\hline \multicolumn{2}{|c|}{ Dominance } & \multirow[b]{2}{*}{$250-700$} & \multirow[b]{2}{*}{$350-500$} & \multirow[b]{2}{*}{$250-2688$} & \multirow[b]{2}{*}{$350-1920$} \\
\hline A & B & & & & \\
\hline \multirow[t]{6}{*}{ PD } & VD5 & $0(0)$ & $0(0)$ & $0(0)$ & $0(0)$ \\
\hline & VD10 & $4.8(5.8)$ & $10.4(15.4)$ & $0.9(1.3)$ & $1.7(1.9)$ \\
\hline & VD15 & $20.3(15.2)$ & $23.2(18.5)$ & $10.5(8.1)$ & $14.9(7.2)$ \\
\hline & VD20 & $19.6(14.3)$ & $26.6(20.2)$ & $20.2(11.8)$ & $21.7(10.7)$ \\
\hline & VD25 & $20.7(14.2)$ & $24.7(16.3)$ & $20.8(10.0)$ & $22.8(12.5)$ \\
\hline & VD30 & $24.9(23.7)$ & $24.5(18.8)$ & $24.0(11.3)$ & $28.2(17.4)$ \\
\hline VD5 & PD & 78.1(7.4) & $77.3(6.6)$ & $81.1(5.9)$ & $73.7(8.3)$ \\
\hline VD10 & & $52.8(18.4)$ & $48.4(22.8)$ & $68.8(9.4)$ & $61.8(12.7)$ \\
\hline VD15 & & $30.9(21.4)$ & $31.4(22.2)$ & $39.6(13.7)$ & $33.6(11.6)$ \\
\hline VD20 & & $30.5(17.0)$ & $27.7(20.1)$ & $29.9(13.7)$ & $26.2(11.1)$ \\
\hline VD25 & & $28.9(16.3)$ & $27.6(16.9)$ & $27.2(12.1)$ & $26.3(11.5)$ \\
\hline VD30 & & $35.0(23.8)$ & $30.8(19.7)$ & $24.3(12.4)$ & $26.8(18.0)$ \\
\hline
\end{tabular}

TABLE III

AVERAGE VALUES (STANDARD DEVIATION) OF coverage of two sets FOR NSGA2 $\mathcal{C}(\mathrm{A} \succ \mathrm{B})$

\begin{tabular}{|c|c|c|c|c|c|}
\hline \multicolumn{6}{|c|}{ NSGA2 $\mathcal{C}(\mathrm{A} \succ \mathrm{B})$} \\
\hline \multicolumn{2}{|c|}{ Dominance } & \multirow[b]{2}{*}{250700} & \multirow[b]{2}{*}{350500} & \multirow[b]{2}{*}{2502688} & \multirow[b]{2}{*}{3501920} \\
\hline A & B & & & & \\
\hline PD & VD5 & $0(0)$ & $0(0)$ & $0(0)$ & $0(0)$ \\
\hline & VD10 & $0.9(2.1)$ & $0.9(1.7)$ & $0.1(0.2)$ & $0.3(1.0)$ \\
\hline & VD15 & $8.9(11.8)$ & $13.5(10.9)$ & $5.1(6.0)$ & $4.9(6.5)$ \\
\hline & VD20 & $17.3(13.5)$ & $15.3(18.4)$ & $9.3(10.1)$ & $10.5(10.7)$ \\
\hline & VD25 & $20.7(23.3)$ & $49.4(40.6)$ & $14.1(10.1)$ & $14.2(8.7)$ \\
\hline & VD30 & $78.1(37.3)$ & $94.0(22.8)$ & $14.3(8.0)$ & $46.1(39.5)$ \\
\hline VD5 & PD & $83.1(7.7)$ & $84.0(7.2)$ & $82.3(5.2)$ & 78.7(12.6) \\
\hline VD10 & & $51.6(21.0)$ & $51.6(16.6)$ & $60.2(17.4)$ & $53.5(19.1)$ \\
\hline VD15 & & $25.6(17.9)$ & $23.7(16.7)$ & $27.9(14.2)$ & 29.1(16.6) \\
\hline VD20 & & $16.2(15.5)$ & $22.1(17.4)$ & $20.3(13.0)$ & $22.9(16.9)$ \\
\hline VD25 & & $24.7(24.1)$ & $47.4(42.4)$ & $18.8(9.1)$ & $15.6(11.0)$ \\
\hline VD30 & & $77.8(37.0)$ & $95.4(17.6)$ & $17.6(7.8)$ & $43.7(41.1)$ \\
\hline
\end{tabular}




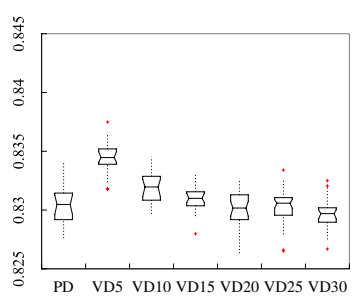

(a) $250-700$

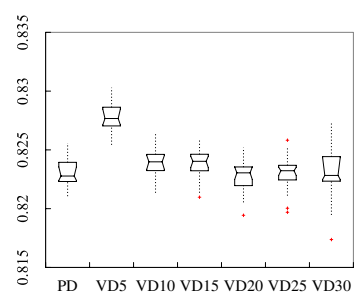

(c) $250-2688$

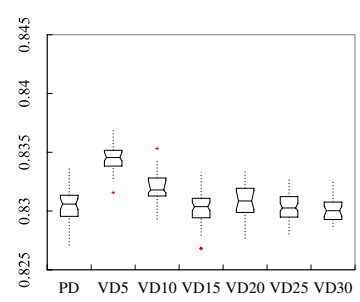

(b) $350-500$

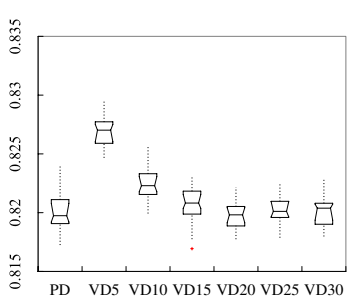

(d) $350-1920$

Fig. 4. Performance of PD and VD on SPEA2 for 4-objective knapsack problem based on size of the space covered metric $\mathcal{S}$. Each graph label indicates the population size - the number of generations

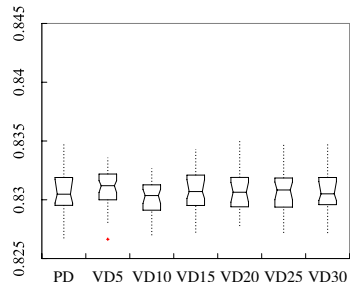

(a) $250-700$

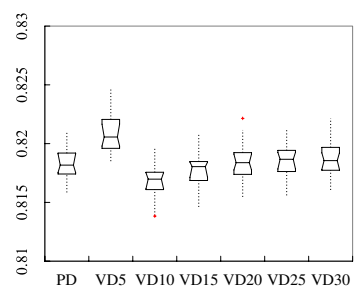

(c) $250-2688$

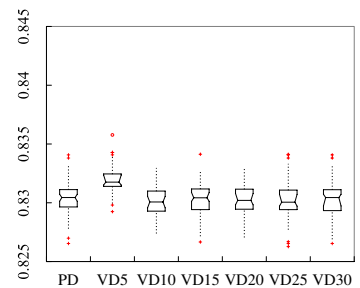

(b) $350-500$

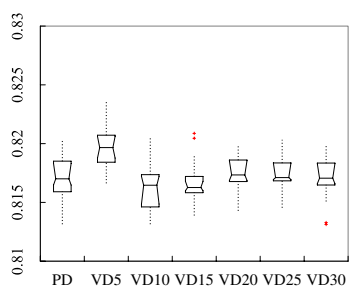

(d) $350-1920$
Fig. 7. Performance of PD and VD on NSGA2 for 4-objective knapsack problem based on size of the space covered metric $\mathcal{S}$. Each graph label indicates the population size - the number of generations

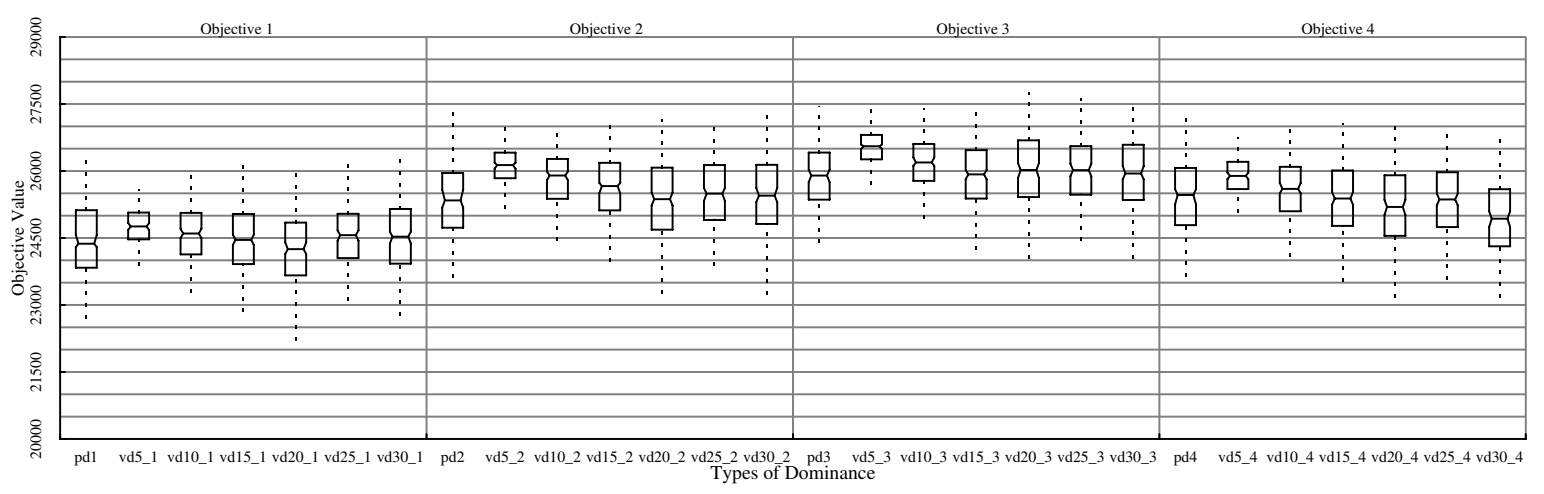

Fig. 5. The distribution of objective values obtained when using conventional Pareto dominance (PD) and volume dominance (VD) on SPEA2 for 4-objective knapsack problem

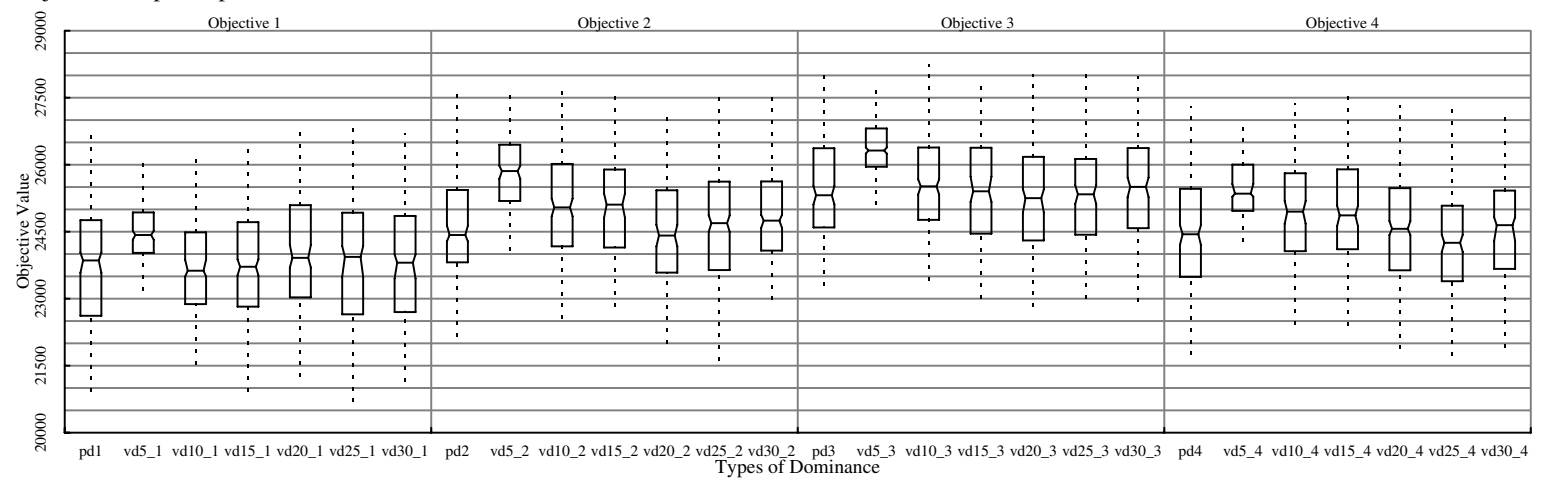

Fig. 6. The distribution of objective values obtained when using conventional Pareto dominance (PD) and volume dominance (VD) on NSGA2 for 4-objective knapsack problem 


\section{FinAl REMARKS}

In this paper we proposed volume dominance, a new form of relaxed Pareto dominance, which is based on comparing the dominated volume of solutions in order to establish preference. We carried out extensive experiments to compare the performance of our proposed volume dominance and Pareto dominance when using three well-known MOEAs namely SEAMO2, SPEA2, and NSGA2. Our results show that for the 4-objective knapsack problem with 750 items, the performance of our volume dominance using high ratios $r S V$ is quite similar to that of Pareto dominance. However, we obtained promising results by using lower ratios $r S V$. Volume dominance using low ratios $r S V$ is capable of obtaining smoother trade-off fronts and is also able to 'push' the tradeoff front in a more uniform manner than the conventional Pareto dominance, i.e. the trade-off front converges as a whole without bias on a particular objective.

We believe that volume dominance provides an alternative approach to assign fitness to solutions in a multiobjective context. Our results suggest that volume dominance can be regarded as more robust than Pareto dominance because it helps the three algorithms implemented here to shown more consistent performance. By adjusting the $r S V$ ratio, users could obtain results driven by different criteria, such as a better coverage, a better size of space covered or a better distribution of the objective values. Future work contemplates comparing different forms of relaxed Pareto dominance in the literature (e.g. $\epsilon$-dominance, $\alpha$-dominance, etc.) using SEAMO2, SPEA2, NSGA2 on the multiple knapsack problem and perhaps other constrained combinatorial optimisation problems. Designing a strategy to automatically adjust the $r S V$ ratio during the search process might further improve the performance of volume dominance. Finally, we believe that our proposed volume dominance is relatively simple to implement but efficient. This new form of relaxed Pareto dominance is worth of further investigation.

\section{REFERENCES}

[1] C.A. Coello Coello, D.A. Van Veldhuizen, and G.B. Lamont. Evolutionary Algorithms for Solving Multi-objective problems, Kluwer academic publishers, 2002.

[2] K. Deb, M. Mohan, and S. Mishra. "Towards a Quick Computation of Well-spread Pareto-optimal Solutions", Proceedings of The 2nd International Conference on Evolutionary Multi-criterion Optimization (EMO 2003), Lecture notes in computer science 2632, pp. 222-236, Springer, 2003.

[3] J.D. Landa-Silva and E.K. Burke. "Using Diversity to Guide the Search in Multi-Objective Optimization", in Carlos A. Coello Coello, Gary B. Lamont (eds.), Applications of Multi-Objective Evolutionary Algorithms, Advances in Natural Computation, Vol. 1, pp. 727-751, World Scientific, 2004.
[4] M. Laumanns, L. Thiele, K. Deb, and E. Zitzler. "Combining Convergence and Diversity in Evolutionary Multi-Objective Optimization", Evolution Computation, 10(3), pp. 263-282, 2002.

[5] M. Koppen, R. Vicente-Garcia, and B. Nickolay. "Fuzzy-ParetoDominance and its Application in Evolutionary Multi-Objective Optimization", Proceedings of The 3rd International Conference on Evolutionary Multi-criterion Optimization (EMO 2005), Lecture notes in computer science 3410, pp. 399-412, Springer, 2005.

[6] E.K. Burke and J.D. Landa-Silva. "The Influence of The Fitness Evaluation Method on The Performance of Multiobjective Search Algorithms", European Journal of Operational Research, 169(3), pp. 875-897, 2006.

[7] V. Pareto. Cours D'Economie Polotique. F.Rouge, Lausanne, 1896

[8] P. Dasgusta, P.P. Chakrabarti, and S.C. DeSarkar. Multiobjective Heuristic Search: An Introduction to Intelligent Search Methods for Multicriteria Optimization, Computational Intelligence, Springer, 1999.

[9] P.L. Yu. "Cone Convexity, Cone Extreme Points, and Nondominated Solutions in Decision Problems with Multiobjectives", Journal of Optimization Theory and Applications, 14(3), pp. 319-377, 1974.

[10] I. Kokolo, K. Hajime, and K. Shigenobu. "Failure of Pareto-based MOEAS: Dose Non-dominated Really Mean near to Optimal?" Proceedings of The 2001 Congress on Evolutionary Computation (CEC 2001), pp. 957-962, IEEE, 2001.

[11] H. Jin and M.L. Wong. "Adaptive Diversity Maintenance and Convergence Guarantee in Multiobjective Evolutionary Algorithms", Proceedings of The 2003 Congress on Evolutionary Computation (CEC 2003), pp, 2498-2505, IEEE, 2003.

[12] J. Peng, H.M. Mok, and W.M. Tse. "Fuzzy Dominance Based on Credibility Distributions", In Fuzzy Systems and Knowledge Discovery Lecture notes in computer science 3613, pp. 295-303, Springer, 2005.

[13] K.N. Le and D. Landa-Silva. "Simple Evolutionary Algorithm with Self-Adaptation for Multi-Objective Optimisation", Submitted for Publication.

[14] C. Mumford. "Simple Population Replacement Strategies for a Steady-State Multi-objective Evolutionary Algorithm", Proceedings of Genetic and Evolutionary Computation (GECCO 2004), Lecture note in computer science 3102, pp. 1389-1400, Springer, 2004.

[15] E. Zitzler, M. Laumanns, and L. Thiele. "SPEA2: Improving the Strength Pareto Evolutionary Algorithm for Multiobjective Optimization", Evolutionary Methods for Design, Optimisation and Control, pp. 95-100, 2002.

[16] K. Deb, A. Pratap, S. Agarwal, and T. Meyarivan. "A Fast and Elitist Multiobjective Genetic Algorithm: NSGA-II", IEEE Transactions on Evolutionary Computation, 6(2), pp. 182-197, IEEE, 2002.

[17] E. Zitzler and L. Thiele. "Multiobjective Evolutionary Algorithms: A Comparative Case Study and the Strength Pareto Approach", IEEE Transactions on Evolutionary Computation, 3(4), pp. 57-271, IEEE, 1999.

[18] S. Bleuler, M. Laumanns, L. Thiele, and E. Zitzler. "PISA - A Platform and Programming Language Independent Interface for Search Algorithms", Proceedings of The 2nd International Conference on Evolutionary Multi-criterion Optimization (EMO 2003), Lecture notes in computer science 2632, pp. 494-508, Springer, 2003.

[19] S. Mostaghim and J. Teich. "The Role of $\epsilon$-dominance in Multi Objective Particle Swarm Optimization Methods", Proceedings of The 2003 Congress on Evolutionary Computation (CEC 2003), pp, 17641771, IEEE, 2003. 